\title{
Hyperplanes and projective embeddings of dual polar spaces
}

\author{
Bart De Bruyn
}

Dedicated to the memory of Adriano Barlotti

\begin{abstract}
This paper is based on the invited talk of the author at the Combinatorics 2010 conference which was held in Verbania (Italy) from June 27th till July 3rd 2010. It discusses hyperplanes and full projective embeddings of dual polar spaces, as well as some of their mutual connections. Many of the discussed results are very recent.
\end{abstract}

Mathematics Subject Classification (2010). Primary 51A45, 51A50; Secondary 05B25.

Keywords. Dual polar space, hyperplane, projective embedding.

\section{Overview}

This paper is based on the invited talk of the author at the Combinatorics 2010 conference which was held in Verbania (Italy) from June 27th till July 3rd 2010. It discusses hyperplanes and full projective embeddings of dual polar spaces, as well as some of their mutual connections. Many of the discussed results are very recent.

In Section 2 of this paper, we give the basic notions and properties regarding (dual) polar spaces which are necessary to understand the actual results which will be discussed in Sections 3 and 4. In Section 2, we also describe four classes of polar spaces (symplectic, parabolic, elliptic, Hermitian) whose corresponding dual polar spaces will play a role in this paper.

In Section 3, we discuss hyperplanes of dual polar spaces. Several classes of hyperplanes of dual polar spaces have been described in the literature. After giving a general introduction to the topic of hyperplanes of dual polar spaces in Section 3.1, we will mainly restrict to one such class of hyperplanes, namely the class of the so-called SDPS-hyperplanes. This class of hyperplanes 
is interesting in the sense that every thick dual polar space is a priori a good candidate for admitting such hyperplanes. The other constructions of hyperplanes of dual polar spaces described in the literature usually only work for specific classes of dual polar spaces, like the symplectic dual polar spaces or the Hermitian dual polar spaces.

In Section 4 we discuss full projective embeddings of dual polar spaces and demonstrate the connection with hyperplanes. Among the various classes of full projective embeddings of dual polar spaces, there are two interesting ones, namely the absolutely universal embeddings and the minimal full polarized embeddings. We mainly discuss these embeddings. For the four classes of dual polar spaces described in Section 2, we discuss what their absolutely universal and minimal full polarized embedding is.

\section{Basic notions and properties regarding (dual) polar spaces}

\subsection{Polar spaces}

A polar space of rank $n \geq 0$ is a pair $(P, \mathcal{S})$ consisting of a set $P$, whose elements are called points, together with a collection $\mathcal{S}$ of subsets of $P$, called singular subspaces, satisfying the following four axioms.

(P1) If $L$ is a singular subspace, then the singular subspaces contained in $L$ are the subspaces of a projective space $\Sigma_{L}$ of dimension $d \in\{-1,0, \ldots$, $n-1\}$. The number $d$ is called the dimension of $L$.

(P2) The intersection of any two singular subspaces is again a singular subspace.

(P3) If $L$ is an $(n-1)$-dimensional singular subspace and if $p \in P \backslash L$, then there exists a unique $(n-1)$-dimensional singular subspace $M$ containing $p$ and intersecting $L$ in an $(n-2)$-dimensional singular subspace. The intersection $M \cap L$ consists of those points of $L$ which are contained in a one-dimensional singular subspace together with $p$.

(P4) There exist two disjoint singular subspaces of dimension $n-1$.

Singular subspaces of dimension $1, n-2$ and $n-1$ are respectively called lines, next-to-maximal singular subspaces and maximal singular subspaces. The polar space $(P, \mathcal{S})$ is called thick if every line contains at least three points and if every next-to-maximal singular subspace is contained in at least three maximal singular subspaces.

If $n=0$, then $P=\emptyset$ and $\mathcal{S}=\{\emptyset\}$. If $n=1$, then $P$ is a set of size at least two and $S=\{\emptyset\} \cup \bigcup_{p \in P}\{\{p\}\}$. If $(P, \mathcal{S})$ is a polar space of rank 2 , then the partial linear space $(\mathcal{P}, \mathcal{L}, \mathrm{I})$, where $\mathcal{L}$ is the set of all lines of $(P, \mathcal{S})$ and $\mathrm{I} \subseteq \mathcal{P} \times \mathcal{L}$ is the incidence relation defined by inclusion, is a generalized quadrangle. A generalized quadrangle a partial linear space which satisfies the following two properties: 
(1) There exist two disjoint lines.

(2) For every line $L$ and every point $p$ not incident with $L$, there exists a unique point $q$ on $L$ collinear with $p$.

The definition of polar space as given above is due to Jacques Tits [37, Chapter 7]. A classification of polar spaces of rank at least three can also be found in Tits [37]. In the remainder of this subsection, we give a description of those polar spaces which will play a role in the present paper.

Let $V$ be a vector space of dimension $2 n \geq 4$ over a field $\mathbb{F}$ which is equipped with a nondegenerate alternating bilinear form $f$. Let $\zeta$ denote the symplectic polarity of the projective space $\mathrm{PG}(V)$ associated with $f$, let $P$ denote the point-set of $\mathrm{PG}(V)$ and let $\mathcal{S}$ be the set of all subspaces $\pi$ of $\mathrm{PG}(V)$ for which $\pi \subseteq \pi^{\zeta}$. Then $(P, \mathcal{S})$ is a polar space of rank $n$. It is called a symplectic polar space and is denoted by $W(2 n-1, \mathbb{F})$.

Suppose $\mathcal{F}$ is a nonsingular quadric or Hermitian variety of Witt index $n \geq 2$ in a projective space $\Sigma$ over a field $\mathbb{F}$. Then the points and subspaces of $\Sigma$ contained in $\mathcal{F}$ define a polar space $\mathcal{P}_{\mathcal{F}}$ of rank $n$. We describe three classes of polar spaces which can be obtained in this way.

(1) Suppose $\mathcal{F}$ is a nonsingular quadric of Witt index $n \geq 2$ in the projective space $\operatorname{PG}(2 n, \mathbb{F})$. Then with respect to some reference system of $\operatorname{PG}(2 n, \mathbb{F}), \mathcal{F}$ has equation $X_{0}^{2}+X_{1} X_{2}+\cdots+X_{2 n-1} X_{2 n}=0$. The associated polar space $\mathcal{P}_{\mathcal{F}}$ is called a parabolic polar space and is denoted by $Q(2 n, \mathbb{F})$. We have $Q(2 n, \mathbb{F}) \cong W(2 n-1, \mathbb{F})$ if and only if $\mathbb{F}$ is a perfect field of characteristic 2, see e.g. De Bruyn \& Pasini [26, Corollary 1.3] and Van Maldeghem [38, Proposition 3.4.13].

(2) Suppose $\mathbb{F}$ and $\mathbb{F}^{\prime}$ are two fields such that $\mathbb{F}^{\prime}$ is a quadratic separable (and hence also Galois) extension of $\mathbb{F}$. Let $\mathcal{F}$ be a nonsingular quadric of Witt index $n$ of $\mathrm{PG}(2 n+1, \mathbb{F})$ which becomes a nonsingular quadric of Witt index $n+1$ of $\mathrm{PG}\left(2 n+1, \mathbb{F}^{\prime}\right)$ when its equation is regarded over the field $\mathbb{F}^{\prime}$. Then with respect to some reference system of $\mathrm{PG}(2 n+1, \mathbb{F}), \mathcal{F}$ has an equation of the form $X_{0}^{2}+X_{0} X_{1}+\delta \cdot X_{1}^{2}+X_{2} X_{3}+\cdots+X_{2 n} X_{2 n+1}=0$, where $\delta \in \mathbb{F}$ is such that the two (necessarily distinct) roots of the polynomial $X^{2}+X+\delta \in \mathbb{F}[X]$ belong to $\mathbb{F}^{\prime} \backslash \mathbb{F}$. The associated polar space $\mathcal{P}_{F}$ is called an elliptic polar space and is denoted by $Q^{-}(2 n+1, \mathbb{F})$.

(3) Suppose $\mathbb{F}$ and $\mathbb{F}^{\prime}$ are two fields such that $\mathbb{F}^{\prime}$ is a quadratic separable (and hence also Galois) extension of $\mathbb{F}$. We denote by $\theta$ the unique nontrivial automorphism of $\mathbb{F}^{\prime}$ fixing each element of $\mathbb{F}$. Let $\mathcal{F}$ be a nonsingular $\theta$ Hermitian variety of Witt index $n$ in $\operatorname{PG}\left(2 n-1, \mathbb{F}^{\prime}\right)$. Then with respect to some reference system of $\mathrm{PG}\left(2 n-1, \mathbb{F}^{\prime}\right), \mathcal{F}$ is given by the equation $\left(X_{0} X_{1}^{\theta}-\right.$ $\left.X_{1} X_{0}^{\theta}\right)+\cdots+\left(X_{2 n-2} X_{2 n-1}^{\theta}-X_{2 n-1} X_{2 n-2}^{\theta}\right)=0$. The associated polar space $\mathcal{P}_{\mathcal{F}}$ is called a Hermitian polar space and is denoted by $H\left(2 n-1, \mathbb{F}^{\prime} / \mathbb{F}\right)$.

In the finite case, we will use slightly different notations for the polar spaces. We will just replace the field by its order. This is illustrated in the following 
table, where we have used the convention that $\mathbb{F}_{q}$ denotes the finite field with $q$ elements.

\begin{tabular}{|c|c|}
\hline$W\left(2 n-1, \mathbb{F}_{q}\right)$ & $W(2 n-1, q)$ \\
\hline$Q\left(2 n, \mathbb{F}_{q}\right)$ & $Q(2 n, q)$ \\
\hline$Q^{-}\left(2 n+1, \mathbb{F}_{q}\right)$ & $Q^{-}(2 n+1, q)$ \\
\hline$H\left(2 n-1, \mathbb{F}_{q^{2}} / \mathbb{F}_{q}\right)$ & $H\left(2 n-1, q^{2}\right)$ \\
\hline
\end{tabular}

\subsection{Dual polar spaces}

Suppose $\Pi$ is a polar space of rank $n \geq 0$. With $\Pi$, there is associated a partial linear space $\Delta$ which is called a dual polar space of rank $n$.

- The points of $\Delta$ are the maximal singular subspaces of $\Pi$.

- The lines of $\Delta$ are the next-to-maximal singular subspaces of $\Pi$.

- Incidence is reverse containment.

A dual polar space is called thick if its associated polar space is thick.

A dual polar space of rank 0 is just a point (no lines). The dual polar spaces of rank 1 are precisely the lines and those of rank 2 are precisely the generalized quadrangles. The four classes of polar spaces described in Section 2.1 will give rise to four classes of dual polar spaces. We will denote a dual polar space by putting a "D" in front of the name of the corresponding polar space. E.g., $D H\left(2 n-1, \mathbb{F}^{\prime} / \mathbb{F}\right)$ denotes the Hermitian dual polar space associated with the Hermitian polar space $H\left(2 n-1, \mathbb{F}^{\prime} / \mathbb{F}\right)$.

In the sequel, we always take the convention that distances are measured in the collinearity graph of the geometry. This is the graph whose vertices are the points of the geometry, two distinct points being adjacent whenever they are collinear, i.e. whenever they are incident with the same line. If we adopt this convention, then the following holds.

Proposition 2.1 (Cameron [5, Theorem 1]). Let $\Delta$ be a dual polar space of rank $n \geq 2$. Then:

(1) The maximal distance between two points of $\Delta$ (= the diameter of $\Delta$ ) is equal to $n$.

(2) For every point $x$ and every line $L$, there exists a unique point on $L$ nearest to $x$.

Property (2) of Proposition 2.1 implies that the partial linear space $\Delta$ belongs to the class of the near polygons which were introduced by Shult and Yanushka in [36].

A convex subspace of a partial linear space $\mathcal{S}=(\mathcal{P}, \mathcal{L}, \mathrm{I})$ is a set $X$ of points of $\mathcal{S}$ satisfying the following two properties:

(a) if a line of $\mathcal{S}$ has at least two of its points in $X$, then all the points of that line are contained in $X$; 
(b) every point on a shortest path between two points of $X$ is also contained in $X$.

If $X$ is a convex subspace of $\mathcal{S}$, then the elements of $X$ and the lines of $\mathcal{S}$ which have all their points in $X$ define a subgeometry of $\mathcal{S}$ which we will denote by $\widetilde{X}$.

Suppose again that $\Pi$ is a polar space of rank $n \geq 0$ and that $\Delta$ is its associated dual polar space. We denote by $\mathrm{d}_{\Delta}(\cdot, \cdot)$ the distance function in $\Delta$. Let $k \in\{0,1, \ldots, n\}$. If $\alpha$ is a singular subspace of dimension $n-1-k$ of $\Pi$, then the set of all maximal singular subspaces of $\Pi$ containing $\alpha$ is a convex subspace of diameter $k$ of $\Delta$. Conversely, every convex subspace of diameter $k$ of $\Delta$ is obtained in this way. The convex subspaces of diameter 0 are the singletons and those of diameter 1 are the lines. There is only one convex subspace of diameter $n$, namely the whole set of points. A convex subspace of diameter 2 is called a quad, a convex subspace of diameter 3 is called a hex and a convex subspace of diameter $n-1$ is called a max. Convex subspaces of dual polar spaces satisfy several nice properties. We collect some of them in the following proposition.

Proposition 2.2. Let $\Delta$ be a dual polar space of rank $n \geq 0$. Then every two points of $\Delta$ at distance $k \in\{0,1, \ldots, n\}$ from each other are contained in a unique convex subspace of diameter $k$. Moreover, the following holds for any convex subspace $F$ of diameter $k$.

(1) $\widetilde{F}$ is a dual polar space of rank $k$.

(2) For every point $x$ of $\Delta$, there exists a (necessarily unique) point $\pi_{F}(x) \in$ $F$ such that $d_{\Delta}(x, y)=d_{\Delta}\left(x, \pi_{F}(x)\right)+d_{\Delta}\left(\pi_{F}(x), y\right)$ for every point $y$ of $F$.

(3) The maximal distance from a point of $\Delta$ to $F$ is equal to $n-k$.

The point $\pi_{F}(x)$ mentioned in Proposition 2.2(2) is called the projection of $x$ onto $F$.

Since dual polar spaces of rank 2 are nothing else than generalized quadrangles, Proposition 2.2 implies that every dual polar space of rank $n \geq 3$ has many subgeometries which are generalized quadrangles.

\section{Hyperplanes of dual polar spaces}

\subsection{Generalities}

A hyperplane of a partial linear space is a set of points, distinct from the whole point set, which intersects each line in either a singleton or the whole line.

An ovoid of a partial linear space is a set of points, distinct from the whole point set, which intersects each line in a singleton. Ovoids are examples of hyperplanes. 
Suppose $\Delta$ is a dual polar space of rank $n \geq 0$. By Proposition 2.1 and Proposition 2.2(3), the set of points of $\Delta$ at distance at most $n-1$ from $x$ is a hyperplane of $\Delta$. This hyperplane is called the singular hyperplane of $\Delta$ with deepest point $x$.

There is also a construction which allows to obtain new hyperplanes of dual polar spaces from other ones.

Theorem 3.1 (De Bruyn and Vandecasteele [27, Proposition 1]). Let $\Delta$ be a dual polar space of rank $n$, let $F$ be a convex subspace of diameter $k$ of $\Delta$ and let $G$ be a hyperplane of the dual polar space $\widetilde{F}$. Let $H$ denote the set of all points at distance at most $n-k-1$ from $F$ together with all points $x$ at distance $n-k$ from $F$ for which $\pi_{F}(x) \in G$. Then $H$ is a hyperplane of $\Delta$.

The hyperplane of $\Delta$ defined in Theorem 3.1 is called the extension of $G$. In the special case that $F$ coincides with the whole point set of $\Delta(k=n)$, we have $H=G$ and the extension is called trivial. A hyperplane of $\Delta$ which does not arise as nontrivial extension of another hyperplane is called reduced.

It is possible that a hyperplane of $\Delta$ arises as extension of two distinct hyperplanes of distinct convex subspaces of $\Delta$. However, the following certainly holds.

Theorem 3.2 (De Bruyn [22, Theorem 1.1]). Let $\Delta$ be a dual polar space of rank $n \geq 0$, let $H$ be a hyperplane of $\Delta$, let $F_{i}, i \in\{1,2\}$, be a convex subspace of $\Delta$ and let $G_{i}, i \in\{1,2\}$, be a reduced hyperplane of $\widetilde{F}_{i}$. If $H$ is the extension of the hyperplane $G_{1}$ of $\widetilde{F_{1}}$ and the extension of the hyperplane $G_{2}$ of $\widetilde{F_{2}}$, then $F_{1}=F_{2}$ and $G_{1}=G_{2}$.

\subsection{SDPS-hyperplanes}

Let $\Delta$ be a thick dual polar space of even rank $2 n$. A set $X$ of points of $\Delta$ is called an $S D P S$-set if it satisfies the following five axioms.

(1) No two points of $X$ are collinear in $\Delta$.

(2) If $Q$ is a quad of $\Delta$ containing at least two points of $X$, then $X \cap Q$ is an ovoid of the generalized quadrangle $\widetilde{Q}$.

(3) The point-line geometry $\widetilde{\Delta}$ whose points are the elements of $X$ and whose lines are the quads of $\Delta$ containing at least two points of $X$ (natural incidence) is a dual polar space of rank $n$.

(4) For all $x, y \in X$, we have $\mathrm{d}_{\Delta}(x, y)=2 \cdot \mathrm{d}_{\widetilde{\Delta}}(x, y)$.

(5) If $L$ is a line containing a point of $X$, then $L$ is contained in a (necessarily unique) quad which contains at least two points of $X$.

A dual polar space $\Delta$ of rank 0 has a unique SDPS-set, namely the singleton $\{x\}$ where $x$ is the unique point of $\Delta$. An SDPS-set of a thick dual polar space $\Delta$ of rank 2 is just an ovoid of the generalized quadrangle $\Delta$.

The acronym "SDPS" is an abbreviation of Sub-Dual-Polar-Space and refers to the fact that the partial linear space $\widetilde{\Delta}$ can be regarded as a sub dual polar 
space of $\Delta$. SDPS-sets were introduced by De Bruyn and Vandecasteele [27] (see also De Bruyn [12, Section 5.6.7]) because of their connection with valuations and hyperplanes of dual polar spaces. They also occur in an implicit way (and only restricted to dual polar spaces of rank 4) in a paper of Pralle and Shpectorov [33] where certain nice hyperplanes of dual polar spaces of rank 4 have been studied.

We will not explain the connection between SDPS-sets and hyperplanes. We refer the reader to [27] or [12, Section 5.6.7] for that. The connection between SDPS-sets and hyperplanes of dual polar spaces is explained in the following theorem.

Theorem 3.3 (De Bruyn and Vandecasteele [27], De Bruyn [12]). Let $X$ be an SDPS-set of a thick dual polar space $\Delta$ of rank $2 n$. Then the maximal distance from a point of $\Delta$ to $X$ is equal to $n$. Moreover, the set of points at distance at most $n-1$ from $X$ is a hyperplane of $\Delta$.

The following is an immediate corollary of Theorems 3.1 and 3.3.

Corollary 3.4. Let $F$ be a convex subspace of diameter $2 k$ of a thick dual polar space $\Delta$ of rank $n$ and let $X$ be an SDPS-set of $\widetilde{F}$. Then the set of points of $\Delta$ at distance at most $n-k-1$ from $X$ is a hyperplane of $\Delta$.

Any hyperplane of a thick dual polar space which can be obtained as described in Corollary 3.4 is called an SDPS-hyperplane. SDPS-hyperplanes can be characterized in the following way.

Theorem 3.5 (De Bruyn [13]). Let $\Delta$ be a thick dual polar space of rank $n \geq 3$ and let $H$ be a hyperplane of $\Delta$. Then $H$ is an SDPS-hyperplane if and only if one of the following three possibilities occurs for each hex $F$ of $\Delta$ :

- $F \subseteq H$;

- $F \cap H$ is a singular hyperplane of $\widetilde{F}$;

- $F \cap H$ consists of those points of $F$ at distance at most 1 from an ovoid of a quad $Q \subseteq F$.

A special case of Theorem 3.5 was also proved in Pralle and Shpectorov [33, Theorem 1].

We are now going to discuss some nontrivial examples of SDPS-sets and we are going to restrict to the finite case. Constructions of SDPS-sets of thick dual polar spaces can be found in De Bruyn [18, 23], De Bruyn \& Vandecasteele [27, Section 4] and Pralle \& Shpectorov [33, Section 1.3].

(I) Consider the finite field $\mathbb{F}_{q^{2}}$ with $q^{2}$ elements and let $\mathbb{F}_{q}$ denote the subfield of order $q$ of $\mathbb{F}_{q}$. Let $\delta$ denote an arbitrary element of $\mathbb{F}_{q^{2}} \backslash \mathbb{F}_{q}$. Then $\mathbb{F}_{q^{2}}=$ $\left\{x_{1}+x_{2} \delta \mid x_{1}, x_{2} \in \mathbb{F}_{q}\right\}$. Define $\tau: \mathbb{F}_{q^{2}} \rightarrow \mathbb{F}_{q} ; x_{1}+x_{2} \delta \mapsto x_{1}$. Consider the following bijection $\phi$ between the vector spaces $\mathbb{F}_{q}^{4 n}$ and $\mathbb{F}_{q^{2}}^{2 n}$ :

$$
\phi\left(x_{1}, x_{2}, \ldots, x_{4 n}\right)=\left(x_{1}+\delta x_{2}, \ldots, x_{4 n-1}+\delta x_{4 n}\right) .
$$


Let $\langle\cdot, \cdot\rangle$ be a nondegenerate alternating bilinear form of the vector space $\mathbb{F}_{q^{2}}^{2 n}$. Then $\tau(\langle\phi(\cdot), \phi(\cdot)\rangle)$ is a nondegenerate alternating bilinear form of $\mathbb{F}_{q}^{4 n}$. Let $D W(4 n-1, q)$ denote the symplectic dual polar space associated with this alternating form of $\mathbb{F}_{q}^{4 n}$. If $\alpha$ is a totally isotropic $n$-dimensional subspace of $\mathbb{F}_{q^{2}}^{2 n}$, then $\phi^{-1}(\alpha)$ is a $2 n$-dimensional totally isotropic subspace of $\mathbb{F}_{q}^{4 n}$. The set of all $2 n$-dimensional totally isotropic subspaces of $\mathbb{F}_{q}^{4 n}$ which can be obtained in this way is an SDPS-set of $D W(4 n-1, q)$.

(II) Consider the finite field $\mathbb{F}_{q^{2}}$ with $q^{2}$ elements and let $\mathbb{F}_{q}$ denote the subfield of order $q$ of $\mathbb{F}_{q^{2}}$. Let $\delta$ denote an arbitrary element of $\mathbb{F}_{q^{2}} \backslash \mathbb{F}_{q}$. Consider the following bijection $\phi$ between the vector spaces $\mathbb{F}_{q}^{4 n+2}$ and $\mathbb{F}_{q^{2}}^{2 n+1}$ :

$$
\phi\left(x_{1}, x_{2}, \ldots, x_{4 n+2}\right)=\left(x_{1}+\delta x_{2}, \ldots, x_{4 n+1}+\delta x_{4 n+2}\right) .
$$

Let $\langle\cdot, \cdot\rangle$ be a nondegenerate Hermitian form of the vector space $\mathbb{F}_{q^{2}}^{2 n+1}$. For every $\bar{x} \in \mathbb{F}_{q^{2}}^{2 n+1}$, we define $h(\bar{x}):=\langle\bar{x}, \bar{x}\rangle$ and for every $\bar{x} \in \mathbb{F}_{q}^{4 n+2}$, we define $q(\bar{x}):=\langle\phi(\bar{x}), \phi(\bar{x})\rangle$. The equation $h(\bar{x})=0$, respectively $q(\bar{x})=0$, defines a nonsingular Hermitian variety $H\left(2 n, q^{2}\right)$ in $\mathrm{PG}\left(2 n, q^{2}\right)$, respectively a nonsingular elliptic quadric $Q^{-}(4 n+1, q)$ in $\mathrm{PG}(4 n+1, q)$. With every maximal subspace of $H\left(2 n, q^{2}\right)$, there corresponds (via the map $\phi^{-1}$ ) a maximal subspace of $Q^{-}(4 n+1, q)$. The set of maximal subspaces of $Q^{-}(4 n+1, q)$ which arise in this way is an SDPS-set of $D Q^{-}(4 n+1, q)$.

The following theorem was proved in De Bruyn [12, Theorem 5.31], but its proof relies very much on Theorem 2 of Pralle \& Shpectorov [33] (whose proof itself invoked some nontrivial result of Pasini \& Shpectorov [32]).

Theorem 3.6 ([12, Theorem 5.31], [33, Theorem 2]). Let $\Delta$ be a finite thick dual polar space of even rank $2 n \geq 4$. If $\Delta$ admits an SDPS-set, then $\Delta$ is isomorphic to either $D W(4 n-1, q)$ or $D Q^{-}(4 n+1, q)$ for some prime power $q$.

Regarding the uniqueness of the SDPS-sets, the following can be said.

Theorem 3.7 (De Bruyn [16]). The dual polar space $D W(4 n-1, q), n \geq 2$, has, up to isomorphism, a unique SDPS-set.

Notice that the conclusion in Theorem 3.7 is not valid if $n=1$. For certain values of $q$, the generalized quadrangle $D W(3, q) \cong Q(4, q)$ has nonisomorphic ovoids.

We believe that there is also, up to isomorphism, a unique SDPS-set in the elliptic dual polar space $D Q^{-}(4 n+1, q)$. We already made some progress toward a uniqueness proof. 


\section{Full projective embeddings of dual polar spaces}

\subsection{Generalities}

A full projective embedding of a partial linear space $\mathcal{S}$ is an injective mapping $e$ from the point-set $\mathcal{P}$ of $\mathcal{S}$ to the point-set of a projective space $\Sigma$ satisfying the following two properties:

- the image of $e$ generates $\Sigma$;

- $e$ maps every line of $\mathcal{S}$ to a full line of $\Sigma$.

Two full projective embeddings $e_{1}: \mathcal{S} \rightarrow \Sigma_{1}$ and $e_{2}: \mathcal{S} \rightarrow \Sigma_{2}$ of the same partial linear space $\mathcal{S}$ are called isomorphic $\left(e_{1} \cong e_{2}\right)$ if there exists an isomorphism $\phi$ between the projective spaces $\Sigma_{1}$ and $\Sigma_{2}$ such that $e_{2}=\phi \circ e_{1}$.

A connection between hyperplanes and full projective embeddings of partial linear spaces is given in the following theorem whose proof is straightforward.

Theorem 4.1. Let $e: \mathcal{S} \rightarrow \Sigma$ be a full projective embedding of a partial linear space $\mathcal{S}$ into a projective space $\Sigma$. If $\alpha$ is a hyperplane of $\Sigma$, then the set of all points of $\mathcal{S}$ which are mapped by e into $\alpha$ is a hyperplane of $\mathcal{S}$.

Any hyperplane which can be obtained in the way as described in Theorem 4.1 is said to arise from (the embedding) e. An important question is the following.

Suppose $H$ is a hyperplane (belonging to some family of hyperplanes) of $\mathcal{S}$ and suppose $\mathcal{S}$ admits at least one full projective embedding. Does $H$ then arise from some full projective embedding of $\mathcal{S}$ ?

The above problem has been considered in the literature for several classes of hyperplanes of partial linear spaces. E.g., Ronan [35, Corollary 2] proved that if $\mathcal{S}$ is fully embeddable and every line of $\mathcal{S}$ is incident with precisely three points, then every hyperplane of $\mathcal{S}$ arises from some full projective embedding of $\mathcal{S}$. The following theorem gives an answer to the above question for a large class of SDPS-hyperplanes. This theorem is a consequence of Theorem 3.5 of the present paper, Corollary 1.5 of Cardinali, De Bruyn \& Pasini [7] and Theorem 2 of Pralle \& Shpectorov [33].

Theorem 4.2. Let $\Delta$ be a finite symplectic or elliptic dual polar space, let $F$ be a convex subspace of diameter $2 k \geq 4$ of $\Delta$ and let $X$ be an SDPS-set of $\widetilde{F}$. Then the SDPS-hyperplane of $\Delta$ associated with $X$ arises from some projective embedding of $\Delta$.

The conclusion of Theorem 4.2 is not valid in the case $k=1$ and $X$ is a nonclassical ovoid of the generalized quadrangle $\widetilde{F}$, i.e. an ovoid of $\widetilde{F}$ which does not arise from a full projective embedding of $\widetilde{F}$. 
Let $e: \mathcal{S} \rightarrow \Sigma$ be a full projective embedding of a partial linear space $\mathcal{S}$ and suppose $\alpha$ is a subspace of $\Sigma$ satisfying the following two properties.

(Q1) $e(p) \notin \alpha$ for every point $p$ of $\mathcal{S}$;

$(\mathrm{Q} 2)<\alpha, e\left(p_{1}\right)>\neq<\alpha, e\left(p_{2}\right)>$ for any two distinct points $p_{1}$ and $p_{2}$ of $\mathcal{S}$.

We denote by $\Sigma / \alpha$ the quotient projective space whose points are the subspaces of $\Sigma$ which have $\alpha$ as a hyperplane. Since $\alpha$ satisfies properties (Q1) and (Q2), it is easily verified that the map which associates with each point $x$ of $\mathcal{S}$ the point $\langle\alpha, e(x)>$ of $\Sigma / \alpha$ defines a full projective embedding of $\mathcal{S}$ into $\Sigma / \alpha$. This full projective embedding is called a quotient of $\alpha$.

If $e_{1}$ and $e_{2}$ are two full projective embeddings of the same partial linear space $\mathcal{S}$, then we say that $e_{1} \geq e_{2}$ if $e_{2}$ is isomorphic to a quotient of $e_{1}$.

\subsection{The absolutely universal embedding and the minimal full polarized em- bedding of a fully embeddable thick dual polar space}

By combining some results from Kasikova \& Shult [28, Section 4.6], Ronan [35, Proposition 3] and Tits [37, 8.6], we have:

Theorem 4.3 ([28, 35, 37]). Let $\Delta$ be a fully embeddable thick dual polar space of rank $n \geq 2$. Then there exists up to isomorphism a unique full projective embedding $\tilde{e}$ such that $\tilde{e} \geq$ e for every full projective embedding e of $\Delta$.

The full projective embedding $\tilde{e}$ alluded to in Theorem 4.3 is called the absolutely universal embedding of $\Delta$. Theorem 4.3 implies that all full projective embeddings of a fully embeddable thick dual polar space of rank at least 2 are defined over the same division ring. The following is a corollary of Theorem 4.3 .

Corollary 4.4. Let $\Delta$ be a fully embeddable thick dual polar space of rank $n \geq 2$. Then the hyperplanes of $\Delta$ which arise from some full projective embedding are precisely the hyperplanes of $\Delta$ which arise from the absolutely universal embedding of $\Delta$.

A full embedding of a thick dual polar space is called polarized if every singular hyperplane arises from it. Full polarized embeddings do exist. Their existence is guaranteed by the following result due to Cardinali, De Bruyn and Pasini $[7]$.

Theorem 4.5 ([7, Corollary 1.8]). Let $\Delta$ be a fully embeddable thick dual polar space of rank $n \geq 2$. Then the absolutely universal embedding of $\Delta$ is polarized.

In another paper, Cardinali, De Bruyn and Pasini [6] proved that among all full polarized embeddings there also exists a "minimal one". 
Theorem 4.6 ([6, Theorem 1.4]). Let $\Delta$ be a fully embeddable thick dual polar space of rank $n \geq 2$. Then there exists up to isomorphism a unique full polarized embedding $\bar{e}$ such that $e \geq \bar{e}$ for every full polarized embedding $e$ of $\Delta$.

The full embedding $\bar{e}$ of $\Delta$ alluded to in Theorem 4.6 is called the minimal full polarized embedding of $\Delta$.

We now give two theorems which give information about the structure of the absolutely universal and the minimal full polarized embedding of a given fully embeddable thick dual polar space.

Suppose $\Delta$ is a fully embeddable thick dual polar space of rank $n \geq 2$ and that $F$ is a convex subspace of diameter $k \geq 2$ of $\Delta$. Then $\widetilde{F}$ is a thick dual polar space of rank $k$. Consider the following problem.

Let $e: \Delta \rightarrow \Sigma$ be a full projective embedding of $\Delta$ into a projective space $\Sigma$ and let $e_{F}: \widetilde{F} \rightarrow \Sigma_{F}$ be the full projective embedding of $\widetilde{F}$ induced by $e$, where $\Sigma_{F}$ is a some suitable subspace of $\Sigma$. What kind of embedding is $e_{F}$ ?

The following two theorems give an answer to the above problem in the case $e$ is the absolutely universal embedding or the minimal full polarized embedding of $\Delta$.

Theorem 4.7 (Cardinali, De Bruyn and Pasini [6, Theorem 1.6]). If $e$ is the minimal full polarized embedding of $\Delta$, then $e_{F}$ is isomorphic to the minimal full polarized embedding of $\widetilde{F}$.

Theorem 4.8 (De Bruyn [22, Theorem 1.4]). If e is the absolutely universal embedding of $\Delta$, then $e_{F}$ is isomorphic to the absolutely universal embedding of $\widetilde{F}$.

The proof of Theorem 4.8 given in [22] relies on results about simple connectedness of hyperplane complements obtained in Cardinali, De Bruyn \& Pasini [7] and McInroy \& Shpectorov [31]. Also the extension construction for hyperplanes (see Theorem 3.1) has played a role in the proof.

\subsection{The absolutely universal and the minimal full polarized embedding of some fully embeddable thick dual polar spaces}

We consider now the following problems for a given fully embeddable thick dual polar space $\Delta$ of rank $n \geq 2$.

- What is the absolutely universal embedding of $\Delta$ ?

- What is the minimal full polarized embedding of $\Delta$ ?

Although there are theoretical results guaranteeing the existence of these embeddings (see Theorems 4.3 and 4.6), it is in practice not so easy to see what these embeddings are. In this subsection, we consider the four classes 
of dual polar spaces described in Section 2 and discuss what their absolutely universal embedding and minimal full polarized embedding is. For two of these classes, we will need to invoke the following result due to De Bruyn and Pasini [25].

Theorem 4.9 ([25, Corollary 1.5]). If e is a full polarized embedding of a thick dual polar space $\Delta$ of rank $n \geq 2$ into a projective space $\mathrm{PG}(V)$, then the dimension of the vector space $V$ is at least $2^{n}$. As a consequence, if $\operatorname{dim}(V)=$ $2^{n}$, then $e$ is isomorphic to the minimal full polarized embedding of $\Delta$.

(I) Consider the symplectic dual polar space $D W(2 n-1, \mathbb{F})$ where $\mathbb{F}$ is a field and $n \geq 2$. This dual polar space is known to admit a natural full projective embedding in $\operatorname{PG}(N-1, \mathbb{F})$, where $N=\left(\begin{array}{c}2 n \\ n\end{array}\right)-\left(\begin{array}{c}2 n \\ n-2\end{array}\right)$, see e.g. Brouwer [2, Theorem 1.1], Cooperstein [10, Proposition 5.1] and De Bruyn [14, 17]. This embedding is called the Grassmann embedding of $D W(2 n-1, \mathbb{F})$. The number $N$ is called the vector dimension of the Grassmann embedding.

The following theorem follows from Cooperstein [10, Theorem B] (finite case) and De Bruyn \& Pasini [24, Corollary 1.2] (general case).

Theorem 4.10 ([10, 24]). If $|\mathbb{F}| \geq 3$, then the Grassmann embedding of the dual polar space $D W(2 n-1, \mathbb{F})$ is absolutely universal.

If $n \geq 3$ and $|\mathbb{F}|=2$, then the Grassmann embedding of $D W(2 n-1, \mathbb{F})$ is not absolutely universal. In that case the vector dimension of the absolutely universal embedding was independently determined by Blokhuis \& Brouwer [1] and Li [29].

Theorem 4.11 ([1, 29]). The vector dimension of the absolutely universal embedding of $D W(2 n-1,2), n \geq 2$, is equal to $\frac{\left(2^{n}+1\right)\left(2^{n-1}+1\right)}{3}$.

The following theorem gives necessary and sufficient conditions for the Grassmann embedding of $D W(2 n-1, \mathbb{F}), n \geq 2$, to be isomorphic to the minimal full polarized embedding.

Theorem 4.12 (De Bruyn [19, Corollary 2.1]). The Grassmann embedding of $D W(2 n-1, \mathbb{F})$ is isomorphic to the minimal full polarized embedding of $D W(2 n-1, \mathbb{F})$ if and only if either $\operatorname{char}(\mathbb{F})=0$ or $(\operatorname{char}(\mathbb{F})=p \neq 0$ and $n<2(p-1))$.

The proof in [19] only invokes linear algebra. For another approach which makes use of advanced Lie algebra and representation theory for the symmetric groups, see also Premet and Suprunenko [34].

(II) Consider the Hermitian dual polar space $D H\left(2 n-1, \mathbb{F}^{\prime} / \mathbb{F}\right)$ where $n \geq 2$ and $\mathbb{F}^{\prime}$ is a separable quadratic extension of $\mathbb{F}$. This dual polar space is known to admit a natural full projective embedding in $\mathrm{PG}(N-1, \mathbb{F})$, where $N=\left(\begin{array}{c}2 n \\ n\end{array}\right)$, 
see Cooperstein [9, Proposition 5.1], De Bruyn [15, Theorem 1.2] and De Bruyn [21, Theorem 1.6]. This embedding is called the Grassmann embedding of $\operatorname{DH}\left(2 n-1, \mathbb{F}^{\prime} / \mathbb{F}\right)$.

The minimal full polarized embedding of $D H\left(2 n-1, \mathbb{F}^{\prime} / \mathbb{F}\right)$ can easily be extracted from the discussion in Cardinali, De Bruyn and Pasini [6, Section $4.2]$.

Theorem 4.13 ([6]). The Grassmann embedding of $D H\left(2 n-1, \mathbb{F}^{\prime} / \mathbb{F}\right)$ is isomorphic to the minimal full polarized embedding of $D H\left(2 n-1, \mathbb{F}^{\prime} / \mathbb{F}\right)$.

The following theorem follows from Cooperstein [9, Theorem B] (finite case) and De Bruyn \& Pasini [24, Corollary 1.4] (general case).

Theorem $4.14([9,24])$. If $|\mathbb{F}| \geq 3$, then the Grassmann embedding of $D H(2 n-$ $\left.1, \mathbb{F}^{\prime} / \mathbb{F}\right)$ is absolutely universal.

If $n \geq 3$ and $|\mathbb{F}|=2$, then the Grassmann embedding of $D H\left(2 n-1, \mathbb{F}^{\prime} / \mathbb{F}\right)$ is not absolutely universal. In that case, the vector dimension of the absolutely universal embedding was determined by $\mathrm{Li}[30]$.

Theorem 4.15 ([30]). The vector dimension of the absolutely universal embedding of $D H(2 n-1,4), n \geq 2$, is equal to $\frac{4^{n}+2}{3}$.

(III) Consider the parabolic dual polar space $D Q(2 n, \mathbb{F})$ where $n \geq 2$ and $\mathbb{F}$ is a field. This dual polar space is known to admit a natural full polarized embedding in $\operatorname{PG}(N-1, \mathbb{F})$ where $N=2^{n}$, see Cameron [4, Theorem 5.1], Chevalley [8] and Buekenhout \& Cameron [3, Section 7]. This embedding is called the spin-embedding of $D Q(2 n, \mathbb{F})$. By Theorem 4.9, we immediately have

Theorem 4.16. The spin embedding of $D Q(2 n, \mathbb{F})$ is isomorphic to the minimal full polarized embedding of $D Q(2 n, \mathbb{F})$.

In the case the characteristic of $\mathbb{F}$ is distinct from 2 , the absolutely universal embedding of $D Q(2 n, \mathbb{F})$ was already determined by Wells [39] in 1983.

Theorem 4.17 ([39, Corollary 6$])$. If char $(\mathbb{F}) \neq 2$, then the spin embedding of $D Q(2 n, \mathbb{F})$ is absolutely universal.

If $\operatorname{char}(\mathbb{F})=2$ and $\mathbb{F}$ is perfect, then $D Q(2 n, \mathbb{F}) \cong D W(2 n-1, \mathbb{F})$ and we have already considered that case before. It is still an open problem what the absolutely universal embedding of $D Q(2 n, \mathbb{F})$ is in the case that $\operatorname{char}(\mathbb{F})=2$ and $\mathbb{F}$ is not perfect.

(IV) Finally, consider the elliptic dual polar space $D Q^{-}(2 n+1, \mathbb{F}), n \geq 2$, defined by a separable quadratic extension $\mathbb{F}^{\prime}$ of the field $\mathbb{F}$. This dual polar space is known to admit a natural full polarized embedding in $\operatorname{PG}(N-1, \mathbb{F})$ 
where $N=2^{n}$, see Cooperstein \& Shult [11, Theorem 2.4] and De Bruyn [20, Theorems 1.1 and 1.2]. This embedding is called the spin-embedding of $D Q^{-}(2 n+1, \mathbb{F})$. By Theorem 4.9, we have

Theorem 4.18. The spin-embedding of $D Q^{-}(2 n+1, \mathbb{F})$ is isomorphic to the minimal full polarized embedding of $D Q^{-}(2 n+1, \mathbb{F})$.

The following theorem was proved by Cooperstein and Shult [11, Theorem 2.5] (finite case) and De Bruyn [20, Corollary 1.4] (general case).

Theorem 4.19 ([11, 20]). The spin-embedding of $D Q^{-}(2 n+1, \mathbb{F})$ is absolutely universal.

\section{References}

[1] Blokhuis, A., Brouwer, A.E.: The universal embedding dimension of the binary symplectic dual polar space. Discrete Math. 264, 3-11 (2003).

[2] Brouwer, A.E.: The composition factors of the Weyl modules with fundamental weights for the symplectic group. Preprint (1992).

[3] Buekenhout, F., Cameron, P.J.: Projective and affine geometry over division rings. Handbook of incidence geometry, 27-62, North-Holland, Amsterdam (1995).

[4] Cameron, P.J.: Flat embeddings of near 2n-gons. In: Finite geometries and designs (Proc. Conf., Chelwood Gate, 1980), pp. 61-71, London Math. Soc. Lecture Note Ser. 49, Cambridge Univ. Press, Cambridge-New York (1981).

[5] Cameron, P.J.: Dual polar spaces. Geom. Dedicata 12, 75-85 (1982).

[6] Cardinali, I., De Bruyn, B., Pasini, A.: Minimal full polarized embeddings of dual polar spaces. J. Algebraic Combin. 25, 7-23 (2007).

[7] Cardinali, I., De Bruyn, B., Pasini, A.: On the simple connectedness of hyperplane complements in dual polar spaces. Discrete Math. 309, 294-303 (2009).

[8] Chevalley, C.C.: The algebraic theory of spinors. Columbia University Press, New York (1954).

[9] Cooperstein, B.N.: On the generation of dual polar spaces of unitary type over finite fields. European J. Combin. 18, 849-856 (1997).

[10] Cooperstein, B.N.: On the generation of dual polar spaces of symplectic type over finite fields. J. Combin. Theory Ser. A 83, 221-232 (1998).

[11] Cooperstein, B.N., Shult, E.E.: A note on embedding and generating dual polar spaces. Adv. Geom. 1, 37-48 (2001).

[12] De Bruyn, B.: Near polygons. Frontiers in Mathematics. Birkhäuser, Basel, 2006.

[13] De Bruyn, B.: A characterization of the SDPS-hyperplanes of dual polar spaces. European J. Combin. 28, 705-714 (2007).

[14] De Bruyn, B.: A decomposition of the natural embedding spaces for the symplectic dual polar spaces. Linear Algebra Appl. 426, 462-477 (2007).

[15] De Bruyn, B.: On the Grassmann-embeddings of the Hermitian dual polar spaces. Linear Multilinear Algebra 56, 665-677 (2008). 
[16] De Bruyn, B.: The uniqueness of the SDPS-set of the symplectic dual polar space $D W(4 n-1, q), n \geq 2$. European J. Combin. 30, 911-922 (2009).

[17] De Bruyn, B.: Some subspaces of the $k$-th exterior power of a symplectic vector space. Linear Algebra Appl. 430, 3095-3104 (2009).

[18] De Bruyn, B.: On geometric SDPS-sets of elliptic dual polar spaces. J. Combin. Theory Ser. A 117, 528-544 (2010).

[19] De Bruyn, B.: On the Grassmann modules for the symplectic groups. J. Algebra 324, 218-230 (2010).

[20] De Bruyn, B.: A note on the spin-embedding of the dual polar space $D Q^{-}(2 n+$ $1, \mathbb{K})$. Ars Combin., to appear.

[21] De Bruyn, B.: On the Grassmann modules for the unitary groups. Linear Multilinear Algebra, to appear.

[22] De Bruyn, B.: On extensions of hyperplanes of dual polar spaces. J. Combin. Theory Ser. A, to appear.

[23] De Bruyn, B.: On geometric SDPS-sets of symplectic dual polar spaces. Preprint.

[24] De Bruyn, B., Pasini, A.: Generating symplectic and Hermitian dual polar spaces over arbitrary fields nonisomorphic to $\mathbb{F}_{2}$. Electron. J. Combin. 14, Research Paper 54, 17 pp. (2007).

[25] De Bruyn, B., Pasini, A.: Minimal scattered sets and polarized embeddings of dual polar spaces. European J. Combin. 28, 1890-1909 (2007).

[26] De Bruyn, B., Pasini, A.: On symplectic polar spaces over non-perfect fields of characteristic 2. Linear Multilinear Algebra 57, 567-575 (2009).

[27] De Bruyn, B., Vandecasteele, P.: Valuations and hyperplanes of dual polar spaces. J. Combin. Theory Ser. A 112, 194-211 (2005).

[28] Kasikova, A., Shult, E.E.: Absolute embeddings of point-line geometries. J. Algebra 238, 265-291 (2001).

[29] Li, P.: On the universal embedding of the $\mathrm{Sp}_{2 n}(2)$ dual polar space. J. Combin. Theory Ser. A 94, 100-117 (2001).

[30] Li, P.: On the universal embedding of the $U_{2 n}(2)$ dual polar space. J. Combin. Theory Ser. A 98, 235-252 (2002).

[31] McInroy, J., Shpectorov, S.: On the simple connectedness of hyperplane complements in dual polar spaces. II. Discrete Math. 310, 1381-1388 (2010).

[32] Pasini, A., Shpectorov, S.: Flag-transitive hyperplane complements in classical generalized quadrangles. Bull. Belg. Math. Soc. Simon Stevin 6, 571-587 (1999).

[33] Pralle, H., Shpectorov, S.: The ovoidal hyperplanes of a dual polar space of rank 4. Adv. Geom. 7, 1-17 (2007).

[34] Premet, A.A., Suprunenko, I.D.: The Weyl modules and the irreducible representations of the symplectic group with the fundamental highest weights. Comm. Algebra 11, 1309-1342 (1983).

[35] Ronan, M.A.: Embeddings and hyperplanes of discrete geometries. European J. Combin. 8, 179-185 (1987).

[36] Shult, E.E., Yanushka, A.: Near n-gons and line systems. Geom. Dedicata 9, 1-72 (1980).

[37] Tits, J.: Buildings of spherical type and finite BN-pairs. Lecture Notes in Mathematics 386. Springer-Verlag, Berlin-New York (1974). 
[38] Van Maldeghem, H.: Generalized polygons. Monographs in Mathematics 93. Birkhäuser, Basel (1998).

[39] Wells, A.L. Jr.: Universal projective embeddings of the Grassmannian, half spinor, and dual orthogonal geometries. Quart. J. Math. Oxford Ser. (2) 34, 375-386 (1983).

Bart De Bruyn

Ghent University

Department of Mathematics

Krijgslaan 281 (S22)

B-9000 Gent (Belgium)

e-mail: bdb@cage. ugent. be 